\title{
Mixoma odontogénico: reporte de caso
}

\author{
Christian Farfán-Gutiérrez ${ }^{\star}$, Esp. $_{1}$, Marco Arce Lazo, Msc.
}

${ }_{1}$ Hospital Regional Honorio Delgado, Arequipa, Perú

Recibido: 26 de mayo del 2016 Aprobado: 29 de agosto del 2016

*Autor de correspondencia: Christian Farfán Gutiérrez. Avenida Daniel Alcides Carrión, La Pampilla José Luis Bustamante y Rivero, Arequipa, Perú. Teléfono: (054) 23-1818 / 21-9702 / 23-3812. Correo electrónico: christianjr_88_@hotmail.com

Cómo citar este artículo: Farfán-Gutiérrez C, Arce-Lazo M. Mixoma odontogénico: reporte de caso. Rev Nac Odontol. 2017;13(25):99-105. doi: http://dx.doi.org/10.16925/od.v13i25.1885

Resumen. Introdución: el objetivo fue determinar el tratamiento quirúrgico conservador o radical adecuado para el mixoma odontogénico. Método: el estudio consideró un caso clínico de mixoma odontogénico en el maxilar superior en una paciente mujer adulta, en el que se escogió el tratamiento radical por las dimensiones de la tumoración. Resultados: se evaluó el caso clínico según criterios clínicos, imagenológicos, histopatológicos y según la evidencia científica actual y se determinó el tratamiento quirúrgico de resección en bloque para este caso. Conclusiones: el tratamiento radical, como la resección "en bloque", parece ser el más adecuado para prevenir la recurrencia de la lesión, especialmente en casos de lesiones extensas como en el caso reportado.

Palabras clave: huesos maxilares, maxilar, mixoma, tumores odontogénicos. 


\title{
Odontogenic myxoma: Case report
}

\begin{abstract}
Introduction: The objective was to determine a conservative or radical surgical treatment suitable for the odontogenic myxoma. Method: The study considered a clinical case of odontogenic myxoma in the upper jaw in an adult female patient, for which a radical treatment was chosen due to the size of the tumor. Results: The clinical case was assessed based on clinical, imaging, and histopathological criteria and according to the current scientific evidence. The en bloc resection treatment was determined for this case. Conclusions: Radical treatment, such as en bloc resection, seems to be the most appropriate to prevent recurrence of the tumor, especially in cases of extensive lesions such as the one reported.
\end{abstract}

Keywords: maxillary bones, jaw, myxoma, odontogenic tumors.

\section{Mixoma odontogênico: relato de caso}

Resumo. Introdução: o objetivo foi determinar o tratamento cirúrgico adequado, se o conservador ou o radical, para o mixoma odontogênico. Método: o estudo considerou um caso clínico de mixoma odontogênico no maxilar superior numa paciente mulher adulta, no qual foi escolhido o tratamento radical pelas dimensões da tumoração. Resultados: avaliou-se o caso clínico segundo critérios clínicos, imagenológicos, histopatológicos e segundo a evidência científica atual; além disso, determinou-se o tratamento cirúrgico de retração em bloco para esse caso. Conclusões: o tratamento radical bem como a retração em bloco parecem ser os mais adequados para prevenir a recorrência da lesão, especialmente em casos de lesões extensas como no caso relatado.

Palavras-chaves: maxilar, mixoma, ossos maxilares, tumores odontogênicos. 


\section{Introducción}

El mixoma odontogénico, descrito por primera vez por Thoma y Goldman en 1947, es una neoplasia benigna poco frecuente $(3-6 \%$ de todos los tumores odontogénicos), pero localmente invasiva [1]. Puede ocurrir a cualquier edad, especialmente entre los 10 y 50 años, con una incidencia máxima en la tercera década $[2,3]$. Afecta con más frecuencia la mandíbula (cuerpo, ángulo-rama superior) [1] y en algunos casos está asociado con la ausencia de elementos dentales o dientes incluidos. Hay dos tipos: mixoma central (colocado en el hueso) y mixoma periférico (tejidos blandos). La ejecución de los exámenes radiológicos como la tomografía computarizada es muy útil en la fase de diagnóstico para obtener información sobre el tamaño del tumor, la perforación del hueso cortical y la posible participación de los tejidos blandos [4-6].

Clínicamente asintomático en las primeras etapas, el mixoma se manifiesta más tarde con perforación del hueso y en etapas avanzadas con movilidad y desplazamiento o reabsorción de la raíz del diente. Al estar desprovisto de la capa de cápsula, está mal demarcado y por lo tanto tiende a infiltrarse en los tejidos circundantes. Su comportamiento invasivo puede estar relacionado con la expresión de una metaloproteinasa y componentes de la matriz extracelular de la matriz, en particular el ácido hialurónico, que revelan la capacidad de promover la invasión tumoral [7].

En cuanto al tratamiento, la resección quirúrgica sigue siendo el tratamiento de elección $[8,9]$. Sin embargo, dada su capacidad para infiltrarse en los tejidos circundantes y su porcentaje de recidiva (26\%), si es tratado de forma conservadora, tiende a repetirse fácilmente (también debido a la falta de una cápsula que rodea y delimita). Sin embargo, no hay consenso a la fecha sobre los métodos de tratamiento quirúrgico, que van desde simples curetajes hasta resecciones "en bloque" con grandes márgenes de seguridad que incluye el tejido sano circundante.

El objetivo de este trabajo es presentar un caso de mixoma odontogénico en el maxilar superior, en el que se optó por la resección en bloque.

\section{Presentación de caso}

Paciente femenino de 45 años que refiere que hace dos años notó un aumento de volumen en encía y movilidad de órgano dentario 15, por lo que decidió acudir a odontólogo en consulta privada, quien le realizó exodoncia (diente 15). Después, notó que el incremento de volumen en la encía no disminuyó, al contrario, aumentó de tamaño, pero al no sentir dolor ni molestias le restó importancia. Un mes antes de su ingreso al Servicio de Cirugía Bucal y Maxilofacial del Hospital Regional Honorio Delgado, en Arequipa, notó movilidad del órgano dentario 16, por lo que acudió al hospital de su localidad, donde al evaluarla le indicaron que tenía un tumor en el maxilar y fue referida al hospital para su tratamiento.

A la evaluación extraoral, presenta asimetría facial por aumento de volumen con predominio de región geniana derecha y borramiento de surco nasogeniano; a la evaluación intraoral, se evidencia una lesión tumoral, de bordes no definidos, de consistencia firme, base amplia, no fistulizada, de color de la mucosa oral que se expande desde el fondo de surco vestibular desde distal del órgano dentario 14 hasta diente 17 , movilidad grado II de diente 16 y desplazamiento hacia palatino, diente 17 sin alteración aparente (figura 1).

La radiografía panorámica evidencia una imagen radiolúcida con imágenes radiopacas en su interior compatibles a tabicaciones características que se asemejan a un "panal de abejas" y a una "raqueta de tenis", que compromete tejido óseo de maxilar superior desde la zona apical del diente 13 hasta diente 16, extendiéndose verticalmente en el maxilar superior sin comprometer seno maxilar. A la evaluación tomográfica, observamos en el maxilar superior una imagen isodensa con imágenes hipodensas en el interior compatible con las tabicaciones mencionadas, que solo ha involucrado tejidos duros y que ha desplazado la tabla ósea vestibular compatible con el diagnóstico presuntivo de un tumor odontogénico de compartimiento benigno (figura 2).

Se le practicó una biopsia incisional para la obtención del diagnóstico definitivo, que dio como resultado a la evaluación microscópica una proliferación de células fusiformes y estrelladas distribuidas en sustancia intercelular y escasas fibras de colágeno compatible con mixoma odontogénico. Tras evaluar las dimensiones del tumor, las estructuras anatómicas que compromete y la alta recurrencia del mixoma, se optó por la resección "en bloque" con márgenes de seguridad para el tratamiento definitivo. Después, la paciente acude a sus 

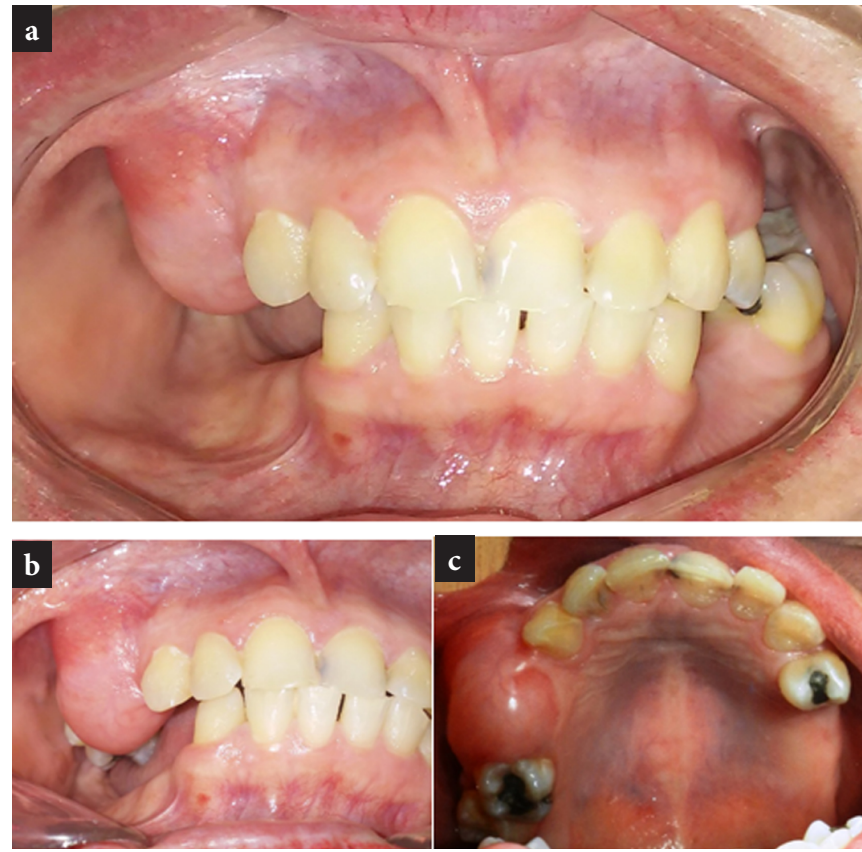

Figura 1 . Imagen intraoral que evidencia lesión tumoral que se extiende desde zona distal de diente 13 hasta mesial de diente 17 , que involucra fondo de surco de primer cuadrante. Nótese que la lesión ha desplazado órgano dentario 17 hacia tabla ósea palatina Fuente: elaboración propia

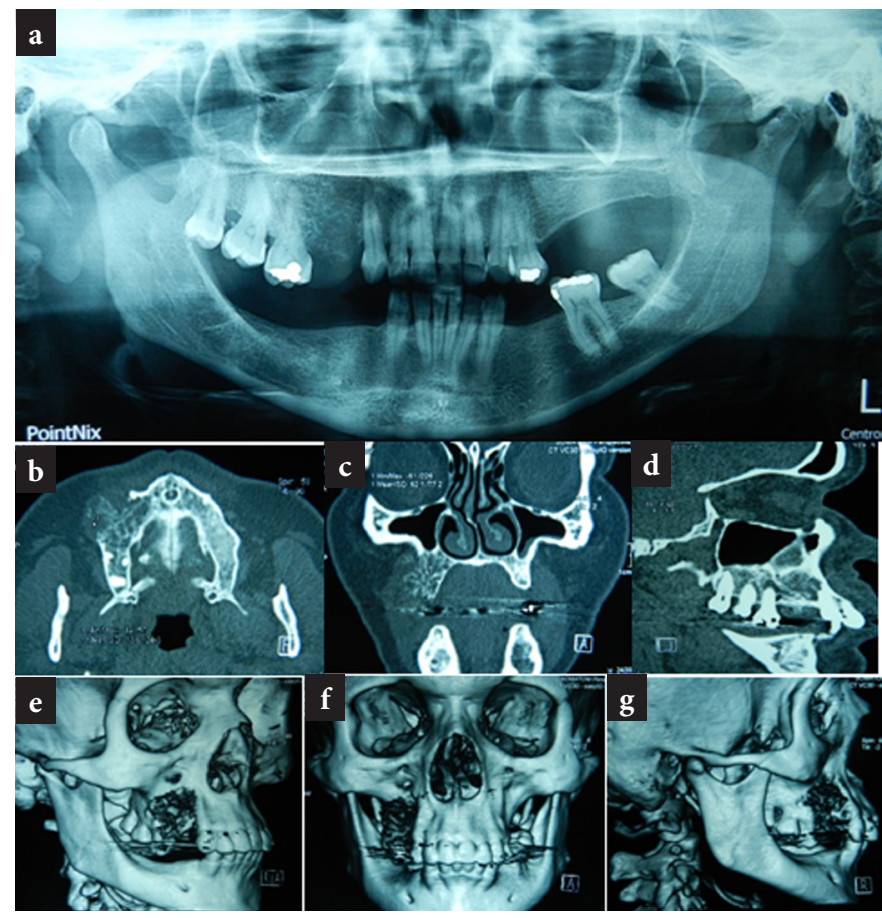

Figura 2. a) Radiografía panorámica que evidencia imagen radiolúcida con tabicaciones que asemejan un "panal de abejas"; b) corte axial, c) corte coronal, d) corte sagital que muestran la dimensión del tumor que involucra parte del maxilar superior (causando la expansión y erosión de la tabla ósea vestibular) y piso de seno maxilar derecho; e), f) y g) reconstrucción 3D que evidencia la erosión ósea causada por el tumor

Fuente: elaboración propia 
controles con normalidad, tiene un seguimiento de doce meses, no se presentó ninguna complicación asociada al tratamiento y continúa actualmente sus controles (figura 3).
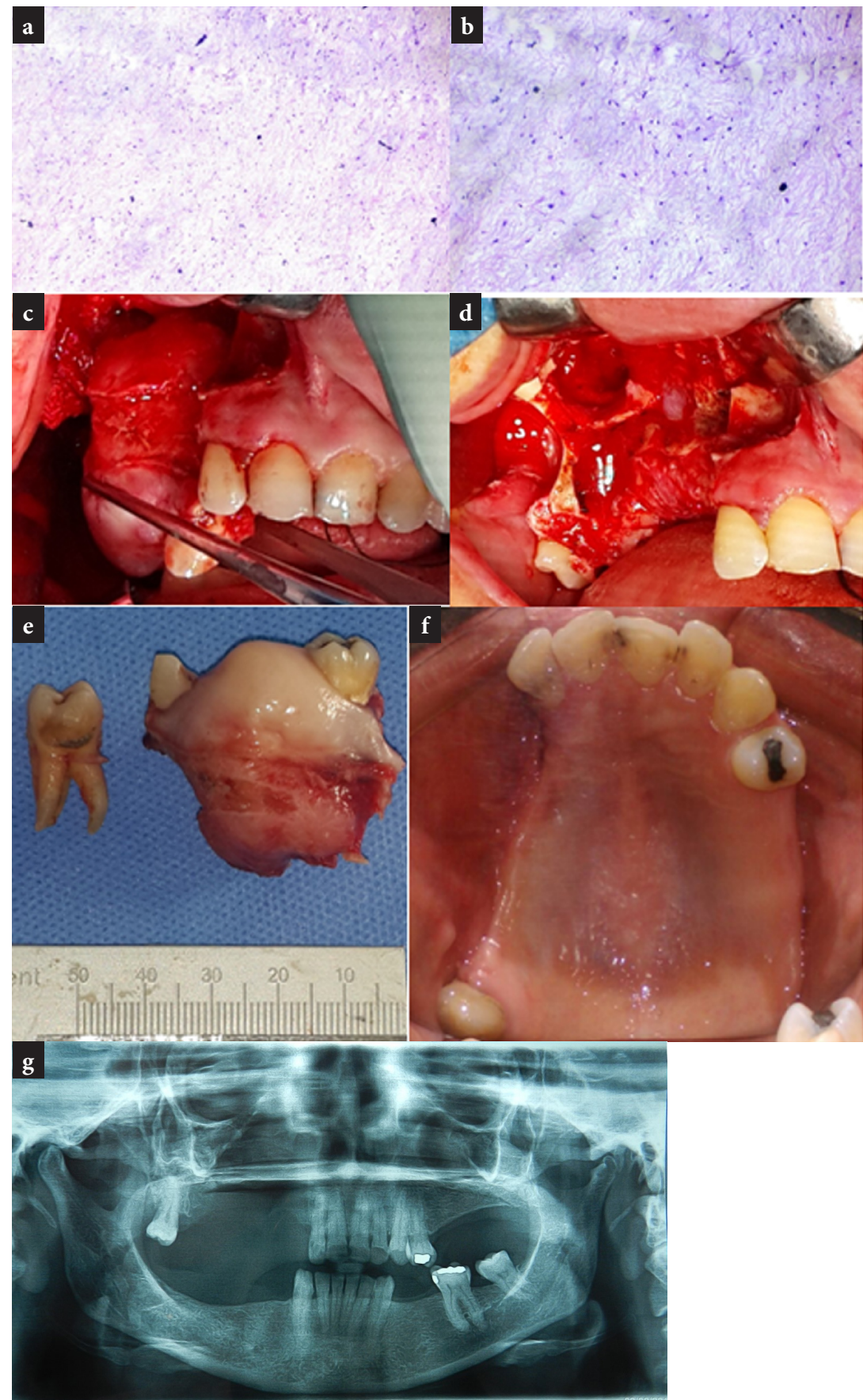

Figura 3. a) Imagen microscópica que evidencia células fusiformes y estrelladas escasas; b) imagen microscópica a mayor aumento donde se ve matriz mixoide, fibras de colágeno y células fusiformes compatibles con mixoma odontogénico; c) y d) resección en bloque de tumoración; e) pieza operatoria con márgenes de seguridad que involucra dientes 14, 16 y 17; f) control posterior de doce meses que muestra la correcta cicatrización y reparación tras la resección; g) radiografía panorámica de control que muestra la gran resección en bloque en el maxilar superior preservando el diente 18 Fuente: elaboración propia 


\section{Discusión}

En la literatura, no hay consenso acerca de los protocolos que deberán utilizarse en el tratamiento de mixoma odontogénico, y los diversos autores proponen opciones de tratamiento con base en su experiencia clínica. La elección del tipo de tratamiento depende de varios factores: la ubicación, el tamaño del tumor, la edad del paciente y la experiencia del cirujano. La enucleación asociada con el curetaje muestra una alta incidencia de recurrencia [9], debido a la consistencia gelatinosa del mixoma y a la ausencia de una cápsula que impida la infiltración de células neoplásicas residuales.

Las directrices quirúrgicas actuales parecen avanzar hacia un tratamiento radical, como la resección "en bloque", que parece ser el más adecuado para prevenir la recurrencia de la lesión, especialmente en casos de lesiones extensas. Esta técnica más radical garantiza la eliminación de todo el bloque del tumor, reduciendo significativamente las recurrencias. Se puede proceder a la inmediata reconstrucción con injertos óseos autólogos o colgajos libres de peroné o cresta ilíaca [1012]. La elección de diferir la fase reconstructiva está motivada por la alta incidencia de recidiva (26\%); esto último podría conducir a la pérdida del injerto y al fracaso del procedimiento, lo que aumenta el costo biológico y la morbilidad de la intervención $[13,14]$.

Cualquiera que sea el tipo de tratamiento quirúrgico, el seguimiento a largo plazo es esencial para el control de las lesiones secundarias, en particular en el caso de tratamiento conservador, en el cual los porcentajes de recidiva son superiores. A pesar de la agresividad y la alta tasa de recurrencia, el pronóstico de mixoma odontogénico, en general, es positivo [15].

\section{Conclusiones}

El tratamiento radical, como la resección "en bloque”, parece ser el más adecuado para prevenir la recurrencia de la lesión, especialmente en casos de lesiones extensas como en el caso reportado.

\section{Confidencialidad de los datos}

Los autores declaran que han seguido los protocolos de su centro de trabajo sobre la publicación de datos de pacientes. Los autores han obtenido el consentimiento informado de los pacientes y/o sujetos referidos en el artículo. Este documento obra en poder del autor de correspondencia. No hubo conflictos de interés.

\section{Referencias}

[1] Thoma KH, Goldman HM. Central mixoma of the jaw. Oral Surg Oral Med Oral Pathol. 1947;33(7): 532-40.

[2] Rotenberg BW, Daniel SJ, Nish IA, Ngan BY, Forte V. Myxomatous lesions of the maxilla in children: A case series and review of management. Int J Pediatr Otorhinolaryngol. 2004;68(10):1251-6.

[3] Keszler A, Domínguez FV, Giannunzio G. Myxoma in childhood: An analysis of 10 cases. J Oral Maxillofac Surg. 1995;53(5):518-21

[4] Barnes L, Eveson JW, Reichart P, Sidransky D. World Health Organization Classification of Tumours. Pathology and genetics of head and neck tumours. Lyon: IARC Press; 2005.

[5] Kawai T, Murakami S, Nishiyama H, Kishino M, Sakuda M, Fuchihata H. Diagnostic imaging for a case of maxillary myxoma with a review of the magnetic resonance images of myxoid lesions. Oral Surg Oral Med Oral Pathol Oral Radiol Endod. 1997;84(4):449-54.

[6] Simon EN, Merkx MA, Vuhahula E, Ngassapa D, Stoelinga PJ. Odontogenic myxoma: A clinicopathological study of 33 cases. Int J Oral Maxillofac Surg. 2004;33(4):333-7.

[7] Miyagi SP, Maranduba CM, Silva F. Dental pulp stem cells express proteins involved in the local invasiveness of odontogenic myxoma. Braz Oral Res. 2012;26(2):139-44.

[8] Boffano P, Gallesio C, Barreca A, Bianchi FA, Garzino-Demo P, Roccia F. Surgical treatment of odontogenic myxoma. J Craniofac Surg. 2011;22(3):982-7.

[9] Buchner A, Merrell PW, Carpenter WM. Relative frequency of central odontogenic tumors: A study of 1,088 cases from Northern California and comparison to studies from other parts of the world. J Oral Maxillofac Surg. 2006;64(9):1343-52.

[10] Chiapasco M, Colletti G, Romeo E, Zaniboni M, Brusati R. Longterm results of mandibular reconstruction with autogenous bone grafts and oral implants after tumor resection. Clin Oral Implants Res. 2008;19(10):1074-80

[11] Chiapasco M, Biglioli F, Autelitano L, Romeo E, Brusati R. Clinical outcome of dental implants placed in fibula-free flaps used for the reconstruction of 
maxillo-mandibular defects following ablation for tumors or osteoradionecrosis. Clin Oral Implants Res. 2006;17(2):220-8.

[12] Chiapasco M, Abati S, Ramundo G, Rossi A, Romeo E, Vogel G. Behavior of implants in bone grafts or free flaps after tumor resection. Clin Oral Implants Res. 2000;11(1):66-75.

[13] Subramanian S, Nastri A, King J, Iseli T. Endoscopic resection of the pterygoid plates following incomplete transoral resection of an odontogenic myxoma. British J Oral and Maxillo Surg. 2016;12(1):22-44.
[14] Santosh K, Mahesh C. An unusually giant myxoma of the maxilla in a child. A case report. Pediatria Polska. 2016;91(5):476-9.

[15] Hammad H, Yousef M, Abd-Albaset M, Abber M, Rima A, Odontogenic myxoma with diffuse calcifications: A case report and review of a rare histologic feature. Oral Surg Oral Med Oral Pathol Oral Radiol Endod. 2016;122(4):116-24. 